\title{
Deterministic Parameter Change Models in Continuous And Discrete Time*
}

\author{
Marcus J. Chambers ${ }^{a}$ and A.M. Robert Taylor $^{b}$ \\ ${ }^{a}$ Department of Economics, University of Essex \\ ${ }^{b}$ Essex Business School, University of Essex
}

November 2018

\begin{abstract}
We consider a model of deterministic one-time parameter change in a continuous time autoregressive model around a deterministic trend function. The exact discrete time analogue model is detailed and compared to corresponding parameter change models adopted in the discrete time literature. The relationships between the parameters in the continuous time model and the discrete time analogue model are also explored. Our results show that the discrete time models used in the literature can be justified by the corresponding continuous time model, with a only a minor modification needed for the (most likely) case where the changepoint does not coincide with one of the discrete time observation points. The implications of our results for a number of extant discrete time models and testing procedures are discussed.
\end{abstract}

Keywords: Parameter change, continuous and discrete time, autoregression, trend break, unit root, persistence change, explosive bubbles.

JEL Classification: C22.

\section{Introduction}

In recent years a wide variety of models for discrete time series data have been proposed in the literature which seek to allow for structural change in the parameters of the model. In this paper, for a relatively simple continuous time first order autoregressive process about a deterministic trend, we demonstrate the impact of one-time deterministic parameter change in the autoregressive parameter, the parameters of the deterministic trend, and the scale factor in the continuous time process on its discrete time analogue.

We derive the discrete time analogue model for the one-time change model in continuous time and show that this takes a similar form to the corresponding one-time change model specified directly in discrete time, with the exception that the parameters of the former additionally vary, relative to

\footnotetext{
${ }^{*}$ We are grateful to the Co-Editor, Steve Leybourne, and two anonymous referees for their helpful and constructive comments on an earlier version. Both authors gratefully acknowledge financial support provided by the Economic and Social Research Council of the United Kingdom under research grant ES/M01147X/1. Authors' E-mail Addresses: mchamb@essex.ac.uk (M. Chambers), robert.taylor@essex.ac.uk (R. Taylor)
} 
their values in both the pre- and post-break regimes, for the first discrete time observation point after the changepoint, unless this coincides with one of the discrete time observation points. This is an important exercise because it is implicitly assumed in the discrete time literature that the underlying parameter change coincides with a discrete time observation point. This assumption is unlikely to hold in practice and our set-up allows us to investigate the consequences of this for the discrete time models. We also explore the relationship between the parameters in the continuous time model and its discrete time analogue. We show that a one-time change in the autoregressive parameter in the continuous time model induces breaks in both the autoregressive parameter and the innovation variance parameter in the discrete time analogue. A one-time change in the autoregressive parameter also induces breaks in the intercept and trend terms in the single equation discrete time analogue model. The implications of these results for a number of extant discrete time models and testing procedures including unit root tests, trend break tests, and bubble detection procedures are discussed.

The remainder of the paper is organised as follows. Section 2 outlines our continuous time model which allows for a one-time deterministic change in its parameters. In section 3 exact discrete time representations are derived for both single-equation (Dickey-Fuller) and components forms. The implications of these results for a variety of associated discrete time estimation and testing procedures are discussed in section 4 . Section 5 concludes. Mathematical proofs are provided in the appendix.

\section{The One-Time Deterministic Change Continuous Time Model}

We consider a scalar random variable, $y(t)$, that satisfies, for $0<t \leq T$, the following components representation in continuous time:

$$
\begin{aligned}
y(t) & =\mu_{0}+\delta_{0} t+\mu_{1} 1_{(t>\tau T)}+\delta_{1} 1_{(t>\tau T)} t+z(t) \\
d z(t) & =\left(\rho_{0}+\rho_{1} 1_{(t>\tau T)}\right) z(t) d t+\left(\sigma_{0}+\sigma_{1} 1_{(t>\tau T)}\right) d B(t)
\end{aligned}
$$

where $1_{(x)}$ is the indicator function that equals one if $x$ is true and equals zero otherwise, $d B(t)$ is the increment (with variance $d t$ ) in a standard Brownian motion process, $0<\tau_{L} \leq \tau \leq \tau_{U}<1$ and $T$ denotes the data span. In this general framework a one-time deterministic change in the values of the parameters of the model occurs at $t=\tau T$ which may therefore affect any or all of the deterministic trend function, the autoregressive parameter and the variance.

Remark 1: The deterministic component specified in (2.1) is the continuous time analogue of the deterministic component specified in Model C of Perron (1989,p.1364), which allows for a change in both the slope and level of the series. The continuous time analogue of the deterministic component specified in Model A of Perron (1989), which allows only for a change in level, obtains setting $\delta_{1}=0$ in (2.1). Finally, the continuous time analogue of the deterministic component specified in Model B of Perron (1989), which allows for a change in the slope of the trend function but with no change in the underlying level, is given by imposing $\mu_{1} \equiv-\delta_{1}(\tau T)$ in (2.1), which is equivalent to replacing $\delta_{1} 1_{(t>\tau T)} t$ in $(2.1)$ by $\delta_{1} 1_{(t>\tau T)}(t-\tau T)$ and setting $\mu_{1}=0$. 
Remark 2: The formulation in (2.1)-(2.2) allows for a one-time change in any or all of the autoregressive, deterministic trend and scale parameters of the continuous time model. The results which follow generalise in an entirely obvious way if we were to allow for multiple such deterministic changes in these parameters. Suppose we allow for a finite number, $m$ say, of such changepoints. Here, rather than the two regimes which occur in the exact discrete time representation given in Theorem 1 we would now obtain $m+1$ such regimes each separated by an interregnum period of the type given in (3.2) wherever the changepoint did not coincide with a discrete time observation point. This would therefore allow, for example, for the possibility that the autoregressive parameter displays a break at a different point in time from a break in the parameters of the deterministic trend function.

Taking the differential of (2.1), substituting for $d z(t)$ using (2.2) and for $z(t)$ using (2.1), results in the following stochastic differential equation for $y(t)$ :

$$
\begin{aligned}
d y(t)= & \left\{\left(\delta_{0}+\delta_{1} 1_{(t>\tau T)}\right)-\left(\rho_{0}+\rho_{1} 1_{(t>\tau T)}\right)\left[\left(\mu_{0}+\mu_{1} 1_{(t>\tau T)}\right)+\left(\delta_{0}+\delta_{1} 1_{(t>\tau T)}\right) t\right]\right. \\
& \left.+\left(\rho_{0}+\rho_{1} 1_{(t>\tau T)}\right) y(t)\right\} d t+\left(\sigma_{0}+\sigma_{1} 1_{(t>\tau T)}\right) d B(t), \quad 0<t \leq T .
\end{aligned}
$$

The two regimes are given by

$$
d y(t)=\left[\pi_{0}+\gamma_{0} t+\rho_{0} y(t)\right] d t+\sigma_{0} d B(t), \quad 0<t \leq \tau T,
$$

where $\pi_{0}:=\delta_{0}-\rho_{0} \mu_{0}$ and $\gamma_{0}:=-\rho_{0} \delta_{0}$, and

$$
d y(t)=\left[\pi_{1}+\gamma_{1} t+\alpha_{1} y(t)\right] d t+\nu_{1} d B(t), \quad \tau T<t \leq T,
$$

where $\pi_{1}:=\delta_{0}+\delta_{1}-\left(\rho_{0}+\rho_{1}\right)\left(\mu_{0}+\mu_{1}\right), \gamma_{1}:=-\left(\rho_{0}+\rho_{1}\right)\left(\delta_{0}+\delta_{1}\right), \alpha_{1}:=\rho_{0}+\rho_{1}$ and $\nu_{1}:=\sigma_{0}+\sigma_{1}$. In what follows we assume that $y(t)$ is a stock variable ${ }^{1}$ such that the observed sequence is obtained at equispaced sampling intervals of length $0<h \leq 1$ resulting in $\left\{y_{t h}=y(t h)\right\}_{t=1}^{N}$. The sample size is $N$ and $N h=T .^{2}$

The continuous time framework allows for the possibility that the changepoint does not coincide with any observation point $t h$ but can lie at some point between two observations at times $t h-h$ and $t h$. While this may be less important for high frequency data it is potentially of value when observations are made less frequently, say monthly or quarterly or even annually. For example, with UK quarterly macroeconomic data, a new government that implements different policies following a general election in the middle of a quarter may affect the model parameters at a point in time which does not coincide with the observed process. The continuous time model defined in (2.1) and (2.2)

\footnotetext{
${ }^{1}$ Qualitatively similar results to those given in this paper for stock variables are also obtained for the case where $y(t)$ is a flow variable; the only change is that the resulting discrete time analogue models will be driven by errors which follow moving average, rather than serially uncorrelated, processes. Furthermore the results concerning quasi-GLS detrending for a stock variable derived in Chambers (2015) would also need appropriate modification for use in unit root testing problems when the variable is a flow.

${ }^{2}$ The results which follow are derived for an arbitrary sampling interval length, $h$. In order to compare the resulting discrete time models that obtain with those used in the extant discrete time literature, which do not take the sampling frequency into account, we may simply set $h=1$ which leads to the usual sample index $t=1, \ldots, T$.
} 
allows for such possibilities.

The solution to (2.3), which is unique in the mean square sense ${ }^{3}$ is given by

$$
\begin{aligned}
y(t)= & \exp \left\{\left(\rho_{0}+\rho_{1} 1_{(t>\tau T)}\right) t\right\} y(0) \\
& +\int_{0}^{t} \exp \left\{\left(\rho_{0}+\rho_{1} 1_{(t>\tau T)}\right)(t-r)\right\}\left\{\left(\delta_{0}+\delta_{1} 1_{(t>\tau T)}\right)\right. \\
& \left.-\left(\rho_{0}+\rho_{1} 1_{(t>\tau T)}\right)\left[\left(\mu_{0}+\mu_{1} 1_{(t>\tau T)}\right)+\left(\delta_{0}+\delta_{1} 1_{(t>\tau T)}\right) r\right]\right\} d r \\
& +\left(\sigma_{0}+\sigma_{1} 1_{(t>\tau T)}\right) \int_{0}^{t} \exp \left\{\left(\rho_{0}+\rho_{1} 1_{(t>\tau T)}\right)(t-r)\right\} d B(r), \quad t>0 .
\end{aligned}
$$

This solution enables the dynamic evolution of $y_{t h}$ in terms of its past values to be determined. It is convenient, in what follows, to assume that $t_{0} h<\tau T<t_{1} h=\left(t_{0}+1\right) h$, i.e. that the changepoint occurs at some point between the observations $t_{0} h$ and $t_{1} h$ where $t_{1}:=\left(t_{0}+1\right)$. We will, however, subsequently consider the specific cases where the changepoint coincides with one of these observation points.

\section{Exact Discrete Time Representation}

In Theorem 1 we now provide the exact discrete time representation in single equation form for the observed process. ${ }^{4}$ The result extends Theorem 2(c) of Bergstrom (1984) to a model with a time trend as well as a breakpoint and different parameters in the two regimes. Corresponding results for the corresponding components form representation will subsequently be discussed in Remarks 5 and 6 .

Theorem 1 Let $y(t)$ be generated by (2.1) and (2.2). Then observations made at equispaced sampling intervals of length $h$ satisfy the following exact discrete time representation:

$$
\begin{aligned}
& y_{t h}=c_{00}+c_{01} t h+\phi_{0} y_{t h-h}+\eta_{0, t h}, \quad t=1, \ldots, t_{0}, \\
& y_{t h}=c_{b 0}+c_{b 1} t h+\phi_{b} y_{t h-h}+\eta_{b, t h}, \quad t=t_{1}, \\
& y_{t h}=c_{10}+c_{11} t h+\phi_{1} y_{t h-h}+\eta_{1, t h}, \quad t=t_{1}+1, \ldots, N,
\end{aligned}
$$

where the autoregressive coefficients are given by $\phi_{0}:=\exp \left\{\rho_{0} h\right\}, \phi_{b}:=\exp \left\{\rho_{0} h+\rho_{1}\left(t_{1} h-\tau T\right)\right\}$ and $\phi_{1}:=\exp \left\{\left(\rho_{0}+\rho_{1}\right) h\right\}$, the intercepts are given by $c_{00}:=h \phi_{0} \delta_{0}+\left(1-\phi_{0}\right) \mu_{0}, c_{b 0}:=h \phi_{b} \delta_{0}+\left(1-\phi_{b}\right) \mu_{0}+$ $\mu_{1}-\exp \left\{\left(\rho_{0}+\rho_{1}\right)\left(t_{1} h-\tau T\right)\right\}\left(\mu_{1}+\delta_{1} \tau T\right)$, and $c_{10}:=h \phi_{1}\left(\delta_{0}+\delta_{1}\right)+\left(1-\phi_{1}\right)\left(\mu_{0}+\mu_{1}\right)$, and the trend parameters are given by $c_{01}:=\left(1-\phi_{0}\right) \delta_{0}, c_{b 1}:=\delta_{1}+\left(1-\phi_{b}\right) \delta_{0}$ and $c_{11}:=\left(1-\phi_{1}\right)\left(\delta_{0}+\delta_{1}\right)$. In addition, the disturbances, $\eta_{0, t h}, \eta_{1, t h}$ and $\eta_{b, t_{1} h}$, are individually and mutually serially uncorrelated

\footnotetext{
${ }^{3}$ The form of the solution follows from a straightforward extension of Theorem 2(a) of Bergstrom (1984), while its uniqueness in mean square follows by extending Theorem 2(b) of Bergstrom (1984) to the present model.

${ }^{4}$ The dependence of the parameters of the discrete time representation on the sampling interval $h$ has been suppressed purely for notational convenience.
} 
with variances given by, respectively,

$$
\omega_{0}^{2}:=\sigma_{0}^{2} \frac{\left(\exp \left\{2 \rho_{0} h\right\}-1\right)}{2 \rho_{0}}, \quad \omega_{1}^{2}:=\left(\sigma_{0}+\sigma_{1}\right)^{2} \frac{\left(\exp \left\{2\left(\rho_{0}+\rho_{1}\right) h\right\}-1\right)}{2\left(\rho_{0}+\rho_{1}\right)},
$$

and

$$
\begin{aligned}
\omega_{b}^{2}:= & \sigma_{0}^{2} \exp \left\{2\left(\rho_{0}+\rho_{1}\right)\left(t_{1} h-\tau T\right)\right\} \frac{\left(\exp \left\{2 \rho_{0}\left(\tau T-t_{0} h\right)\right\}-1\right)}{2 \rho_{0}} \\
& +\left(\sigma_{0}+\sigma_{1}\right)^{2} \frac{\left(\exp \left\{2\left(\rho_{0}+\rho_{1}\right)\left(t_{1} h-\tau T\right)\right\}-1\right)}{2\left(\rho_{0}+\rho_{1}\right)} .
\end{aligned}
$$

Remark 3: It is clear from Theorem 1 that a break in the continuous time autoregressive parameter affects all of the discrete time parameters in (3.1)-(3.3) including the disturbance variance, not just the discrete time autoregressive parameter. Moreover, a break in the continuous time trend parameter affects not only the discrete time trend parameter but also the intercept. In contrast, breaks in the continuous time intercept and scale parameters affect only the discrete time intercept and innovation variance parameters, respectively. In the interregnum interval that contains the break point, $\left(t_{0} h, t_{1} h\right]$, there is an additional term in the intercept in (3.2), arising from the final term in the expression for $c_{b 0}$ in Theorem 1, involving the true break location $\tau T$. This occurs because the parameters governing the evolution of the continuous time process change at this point within the sampling interval and the presence of this additional term captures this feature. Notice that the trend, autoregressive and innovation variance parameters in the interregnum period also differ from the corresponding values of those parameters in the pre- and post-break periods. These observations have implications for the conduct and interpretation of discrete time estimation and inference in cases where parameter breaks are considered, including trend break estimation and testing, unit root testing, and bubble testing, which we will discuss further in section 4.

Remark 4: The model specified in (2.1) and (2.2) does not restrict the sign of the autoregressive coefficients $\rho_{0}$ and $\rho_{0}+\rho_{1}$. The process $y(t)$ is stationary/integrated/explosive according to whether these coefficients are negative/zero/positive, respectively. Zero roots in continuous time translate into unit roots in discrete time as is clearly seen by inspection of $\phi_{0}, \phi_{b}$ and $\phi_{1}$ which are all equal to unity when $\rho_{0}=\rho_{1}=0$ (or $\rho_{0}+\rho_{1}=0$ in the case of $\phi_{1}$ ). In such cases the intercept (or drift) coefficients are such that $c_{00}=h \delta_{0}, c_{b 0}=h \delta_{0}-\delta_{1} \tau T$ and $c_{10}=h\left(\delta_{0}+\delta_{1}\right)$, while the discrete time trend parameters are $c_{01}=c_{11}=0$ and $c_{b 1}=\delta_{1}$. Hence although only a drift term appears in the pre- and post-break periods, a linear trend term appears during the interregnum period of the form $c_{b 0}+c_{b 1} t_{1} h=h \delta_{0}+\delta_{1}\left(t_{1} h-\tau T\right)$. Observe that this value lies between $c_{00}$ and $c_{10}$ in view of the fact that $0 \leq t_{1} h-\tau T \leq h$. Furthermore the variances in the zero/unit root cases can be found by using the series expansion of $\exp \{x\}$ and noting that $(\exp \{h x\}-1) / x=h+O\left(h^{2} x\right)$; this results in $\omega_{0}^{2}=\sigma_{0}^{2} h, \omega_{b}^{2}=\sigma_{0}^{2}\left(\tau T-t_{0} h\right)+\left(\sigma_{0}+\sigma_{1}\right)^{2}\left(t_{1} h-\tau T\right)$ and $\omega_{1}^{2}=\left(\sigma_{0}+\sigma_{1}\right)^{2} h$. Note that, if $\lambda$ denotes the proportion of the interregnum period prior to the break taking place, so that $\tau T-t_{0} h=\lambda h$ and 
$t_{1} h-\tau T=(1-\lambda) h$, then $\omega_{b}^{2}=\lambda \omega_{0}^{2}+(1-\lambda) \omega_{1}^{2}$. Hence with zero/unit roots the variance in the interregnum period is a weighted average of the pre- and post-break variances.

Remark 5: The representations for $y_{t h}$ in the pre- and post-break periods, given in (3.1) and (3.3) respectively, are also consistent with a discrete time components representation. To demonstrate this, evaluating (2.1) at an observation point in the pre-break period yields

$$
y_{t h}=\mu_{0}+\delta_{0} t h+z_{t h}, \quad t=1, \ldots, t_{0},
$$

where $z_{t h}=z(t h)$. However, $z(t h)$ satisfies $(2.2)$ and so its law of motion is given by

$$
z_{t h}=\phi_{0} z_{t h-h}+\eta_{0, t h}, \quad t=1, \ldots, t_{0},
$$

where $\phi_{0}=\exp \left\{\rho_{0} h\right\}$ and $\eta_{0, t h}$ is the disturbance in (3.1). The discrete time components representation comprises (3.4) and (3.5). That it is consistent with (3.1) can be shown by noting from (3.4) that $z_{t h}=y_{t h}-\mu_{0}-\delta_{0} t h$ and then substituting for $z_{t h}$ and its lag in (3.5):

$$
y_{t h}-\mu_{0}-\delta_{0} t h=\phi_{0}\left[y_{t h-h}-\mu_{0}-\delta_{0}(t h-h)\right]+\eta_{0, t h} .
$$

Rearranging results in $y_{t h}=h \phi_{0} \delta_{0}+\left(1-\phi_{0}\right) \mu_{0}+\left(1-\phi_{0}\right) t h+\phi_{0} y_{t h-h}+\eta_{0, t h}$, as required. Similar operations applied to the post-break period yield the discrete time components representation for $t=t_{1}+1, \ldots, N$ :

$$
\begin{aligned}
& y_{t h}=\mu_{0}+\mu_{1}+\left(\delta_{0}+\delta_{1}\right) t h+z_{t h} \\
& z_{t h}=\phi_{1} z_{t h-h}+\eta_{1, t h}
\end{aligned}
$$

this can be shown to be consistent with the single-equation representation for $y_{t h}$ given in (3.3).

Remark 6: It is also possible to consider a components representation for the interregnum period at time $t_{1} h$. In this case the equation for $y_{t_{1} h}$ is obtained from (2.1) directly as

$$
y_{t_{1} h}=\mu_{0}+\mu_{1}+\left(\delta_{0}+\delta_{1}\right) t_{1} h+z_{t_{1} h},
$$

where $z_{t_{1} h}=z\left(t_{1} h\right)$. It is then a matter of relating $z_{t_{1} h}$ to $z_{t_{0} h}$; as in the derivation of (3.2) this can be achieved in two steps, the first of which relates $z_{t_{1} h}$ to $z(\tau T)$ over the post-break part of the interregnum period, the second relating $z(\tau T)$ to $z_{t_{0} h}$ using the pre-break parameters. This gives

$$
\begin{aligned}
z_{t_{1} h} & =\exp \left\{\left(\rho_{0}+\rho_{1}\right)\left(t_{1} h-\tau T\right)\right\} z(\tau T)+\eta_{b 1, t_{1} h}, \\
z(\tau T) & =\exp \left\{\rho_{0}\left(\tau T-t_{0} h\right)\right\} z_{t_{0} h}+\eta_{b 0, \tau T} .
\end{aligned}
$$

Substituting the second expression in the first results in

$$
z_{t_{1} h}=\phi_{b} z_{t_{0} h}+\eta_{b, t_{1} h}
$$


where $\phi_{b}$ is defined in Theorem 1 and $\eta_{b, t_{1} h}=\eta_{b 1, t_{1} h}+\eta_{b 0, \tau T}$ is the same as in (3.2). The components representation for the interregnum period is, therefore, given by (3.8) and (3.9). However, the implication of this representation for the single equation representation of $y_{t_{1} h}$ differs slightly from that in (3.2). Replacing $z_{t_{1} h}=y_{t_{1} h}-\left(\mu_{0}+\mu_{1}\right)-\left(\delta_{0}+\delta_{1}\right) t_{1} h$ and $z_{t_{0} h}=y_{t_{0} h}-\mu_{0}-\delta_{0} t_{0} h$ in (3.9) and rearranging yields

$$
y_{t_{1} h}=\tilde{c}_{b 0}+c_{b 1} t_{1} h+\phi_{b} y_{t_{0} h}+\eta_{b, t_{1} h},
$$

which differs from (3.2) in the intercept term where $\tilde{c}_{b 0}=h \phi_{b} \delta_{0}+\left(1-\phi_{b}\right) \mu_{0}+\mu_{1}$. In fact, the two intercepts are related by $c_{b 0}=\tilde{c}_{b 0}-\exp \left\{\left(\rho_{0}+\rho_{1}\right)\left(t_{1} h-\tau T\right)\right\}\left(\mu_{1}+\delta_{1} \tau T\right)$. The reason for this difference lies in the treatment of the break in trend during the interregnum period. In the single equation approach in Theorem 1 the trend component is present in the formulation when relating $y_{t_{1} h}$ to $y(\tau T)$ and then $y(\tau T)$ to $y_{t_{0} h}$; the additional terms in $c_{b 0}$ arise from the deterministic integrals that appear in these representations. In the components approach the trend terms are only substituted into the expression once $z_{t_{1} h}$ has been related to $z(\tau T)$ and $z(\tau T)$ related to $z_{t_{0} h}$. The same autoregressive coefficient and disturbance arise in both approaches but the different treatment of the linear trend results in a difference in the intercepts. In this sense the components approach does not fully capture the interaction of the trend break and the temporal aggregation over the interregnum period in the way that the single equation approach does. Of course, such matters are not a concern in models formulated directly in discrete time where it is only possible to identify breaks that correspond with the observation points. The continuous time setting allows these breaks to occur and to be identified within the sampling interval.

Remark 7: Following Remark 1 it is also of interest to relate the exact discrete time representation in Theorem 1 to Models A and B in Perron (1989). The pre-break representation is unchanged but there are some differences that arise in the interregnum and post-break periods, as follows:

$$
\begin{array}{ll}
\text { Model A }\left(\delta_{1}=0\right): & c_{b 0}:=h \phi_{b} \delta_{0}+\left(1-\phi_{b}\right) \mu_{0}-\left(\exp \left\{\left(\rho_{0}+\rho_{1}\right)\left(t_{1} h-\tau T\right)\right\}-1\right) \mu_{1}, \\
& c_{10}:=h \phi_{1} \delta_{0}+\left(1-\phi_{1}\right)\left(\mu_{0}+\mu_{1}\right), \\
& c_{b 1}:=\left(1-\phi_{b}\right) \delta_{0}, \quad c_{11}:=\left(1-\phi_{1}\right) \delta_{0} . \\
\text { Model B }\left(\mu_{1}=-\delta_{1} \tau T\right): & c_{b 0}:=h \phi_{b} \delta_{0}+\left(1-\phi_{b}\right) \mu_{0}-\delta_{1} \tau T \\
& c_{10}:=h \phi_{1}\left(\delta_{0}+\delta_{1}\right)+\left(1-\phi_{1}\right)\left(\mu_{0}-\delta_{1} \tau T\right) .
\end{array}
$$

The trend coefficients, $c_{b 1}$ and $c_{11}$, remain unchanged in Model B, as do all the discrete time variances in both models.

Theorem 1 contains an exact discrete time representation in the most general framework where a break occurs within a sampling interval. It is important to demonstrate that it is also valid in the case where no break occurs and in situations where the break location coincides with one of the end points of the affected sampling interval i.e. at $t_{0} h$ or at $t_{1} h$. We deal with these special cases in turn: No break: this occurs when $\mu_{1}=\delta_{1}=\rho_{1}=\sigma_{1}=0$. It is immediate from the definitions that, in this case, $\phi_{1}=\phi_{0}, c_{10}=c_{00}, c_{11}=c_{01}$ and $\omega_{1}^{2}=\omega_{0}^{2}$, and so (3.1) and (3.3) are equivalent. Turning to (3.2), it is also clear that $\phi_{b}=\phi_{0}, c_{b 0}=c_{00}, c_{b 1}=c_{01}$ and $\omega_{b}^{2}=\omega_{0}^{2}$, hence (3.2) is equivalent to (3.1) 
as required. This model then corresponds to that considered in Theorem 2 of Bergstrom (1984) but with the addition of a time trend.

Break at $t_{0} h$ : in this case, $\tau T=t_{0} h$, and so the break occurs at the beginning of the break period. The pre-break equation, (3.1), continuous to hold, as does the post-break equation, (3.3), and so we need to demonstrate that (3.2) is equivalent to (3.3) in this case. We begin by noting that $t_{1} h-\tau T=t_{1} h-t_{0} h=h$ and so $\phi_{b}=\phi_{1}$ follows immediately. The intercept in this case is then

$$
\begin{aligned}
c_{b 0} & =h \phi_{1} \delta_{0}+\left(1-\phi_{1}\right) \mu_{0}+\left(1-\phi_{1}\right) \mu_{1}-\phi_{1} \delta_{1} t_{0} h \\
& =h \phi_{1}\left(\delta_{0}+\delta_{1}\right)+\left(1-\phi_{1}\right)\left(\mu_{0}+\mu_{1}\right)-\phi_{1} \delta_{1} t_{1} h=c_{10}-\phi_{1} \delta_{1} t_{1} h
\end{aligned}
$$

(using $t_{0} h=t_{1} h-h$ ) while the trend coefficient is $c_{b 1}=\delta_{1}+\left(1-\phi_{1}\right) \delta_{0}$. Combining the two terms results in $c_{b 0}+c_{b 1} t_{1} h=c_{10}-\phi_{1} \delta_{1} t_{1} h+\left(\delta_{1}+\left(1-\phi_{1}\right) \delta_{0}\right) t_{1} h=c_{10}+c_{11} t_{1} h$, as required. It is straightforward to show that $\omega_{b}^{2}=\omega_{1}^{2}$ which demonstrates that (3.2) is equivalent to (3.3).

Break at $t_{1} h$ : here, $\tau T=t_{1} h$ and the break occurs at the end of the break period. The pre- and postbreak equations, (3.1) and (3.3), respectively, continue to hold, and so in this case we need to establish that (3.2) is equivalent to (3.1). We note that $t_{1} h-\tau T=0$ and $\tau T-t_{0} h=h$ and it is straightforward to see that $\phi_{b}=\phi_{0}$. The intercept becomes $c_{b 0}=h \phi_{0} \delta_{0}+\left(1-\phi_{0}\right) \mu_{0}-\delta_{1} t_{1} h=c_{00}-\delta_{1} t_{1} h$, while the trend coefficient is $c_{b 1}=\delta_{1}+\left(1-\phi_{0}\right) \delta_{0}$. Combining yields $c_{b 0}+c_{b 1} t_{1} h=c_{00}-\delta_{1} t_{1} h+\left(\delta_{1}+\left(1-\phi_{0}\right) \delta_{0}\right) t_{1} h=$ $c_{00}+c_{01} t_{1} h$, while it also holds that $\omega_{b}^{2}=\omega_{0}^{2}$, as required.

\section{Some Implications for Methods in Discrete Time}

The results in section 3 have important implications for a number of widely used modelling and testing procedures performed on discrete time data. In particular, those relating to unit root tests which allow for breaks in the deterministic trend function and the related issue of robust trend break testing and associated trend break fraction estimation, and the recent literatures relating to change in the autoregressive parameter, most notably tests and detection and dating procedures for persistence change in macroeconomic data and for rational explosive bubbles in financial data.

\subsection{Methods Relating to Trend Breaks}

Perron (1989) shows that an unmodelled broken intercept and/or trend in the data renders standard unit root tests non-similar and heavily biases these tests towards non-rejection of the unit root null when applied to stochastically stationary series. For a known break date in discrete time, Perron (1989) shows that these deficiencies can be resolved using a two-step procedure whereby the levels data are appropriately detrended in the first step. For Models A, B and C of Perron (1989) this entails running, in the second step, an augmented Dickey-Fuller [ADF] test on the residuals from the OLS regression of the observed data $y_{t}, t=1, \ldots, T$, onto $Z_{t}^{i}\left(t_{0}\right), i \in\{A, B, C\}$, where $Z_{t}^{i}\left(t_{0}\right)$ is the set of deterministic regressors implied by either: Model $\mathrm{A}, Z_{t}^{A}\left(t_{0}\right):=\left\{1, t, 1_{\left(t>t_{0}\right)}\right\}$; Model $\mathrm{B}$, 
$Z_{t}^{B}\left(t_{0}\right):=\left\{1, t, 1_{\left(t>t_{0}\right)}\left(t-t_{0}\right)\right\} ;$ Model $\mathrm{C}, Z_{t}^{C}\left(t_{0}\right):=\left\{1, t, 1_{\left(t>t_{0}\right)} 1_{\left(t>t_{0}\right)} t\right\} .{ }^{5}$ Quasi-GLS detrended analogues of this approach are developed in Perron and Rodríguez (2003). Here the first-step is conducted using quasi-GLS, rather than OLS, detrending.

Specialising our results in Theorem 1 and related results in Remarks 5, 6 and 7 to the case where only the intercept and/or trend coefficients can display structural change and setting $h=1$, as in footnote 3 , it is clear from a comparison with the corresponding discrete time models in Perron (1989,p.1364), inter alia, that the two-step approaches developed in the discrete time literature remain appropriate for data obtained by discrete time sampling from the continuous time model in (2.1)-(2.2). This is because although in the single equation representation given in Theorem 1 the interregnum observation at $t=t_{1}$ in (3.2) will have different parameter values on the intercept and trend terms from those which apply in either (3.1) or (3.3) (excepting the case where the changepoint coincides with $t_{0}$ or $t_{1}$ ), this is not the case in the components form discussed in Remarks 5 and 6 .

Where the break date occurs at an unknown point in discrete time, the approaches outlined above have been extended in two separate ways. The first proposed in, inter alia, Zivot and Andrews (1992), performs the approach outlined in Perron (1989) for all possible break dates within a pre-defined set of dates and forms a unit root test based on the most negative of the resulting set of ADF statistics. In contrast to the two-step approach of Perron (1989), however, Zivot and Andrews (1992) include the deterministic variables directly in the ADF regression. As (3.2) shows, this is not appropriate for data obtained by discrete time sampling from (2.1)-(2.2) (unless the breakpoint coincides with an observation point) and the impulse dummy $1_{\left(t=t_{0}+1\right)}$ should be included in the ADF regression.

In the second approach the unknown location of the break in the deterministic trend function is first estimated. An obvious estimator, discussed in Perron and Zhu (2005) and Kim and Perron (2009), is the levels estimator obtained as the location which minimises the sum of squared residuals (SSR) from the OLS regression of $y_{t}$ onto either $Z_{t}^{A}(s), Z_{t}^{B}(s)$ or $Z_{t}^{C}(s)$, according to which of Models A, B and $\mathrm{C}$ is specified, taken over the set of possible break dates $s \in\left\{t_{L}, t_{L}+1, \ldots, t_{U}\right\}$, such that $t_{L}:=\lfloor\pi T\rfloor$ and $t_{U}:=T-\lfloor\pi T\rfloor$, with $\pi \in(0,1)$ a user-defined trimming parameter and $\lfloor\cdot\rfloor$ denoting the integer part of its argument. The corresponding quasi-GLS estimator is considered in Carrion-i-Silvestre et al. (2009). For Model C, a first difference estimator of the trend break location can also be used by estimating the location of a level break in the first differences of the data. The foregoing estimators are all based on static estimation. An alternative estimator, originally proposed in Hatanaka and Yamada (1999) and discussed further in Kim and Perron (1998), minimises the SSR from the dynamic OLS regression of $y_{t}$ onto either $y_{t-1}, Z_{t}^{i}(s)$ and $1_{(t=s+1)}$ for $i \in\{A, C\}$ or onto $y_{t-1}, Z_{t}^{i}(s), 1_{(t=s+1)}$ and $1_{(t \geq s)}$ for $i=B$. In each case, one then proceeds as above but using the estimated break date in place of the true break date. ${ }^{6}$ It should again be clear that the estimators based on static regressions all remain appropriate for data obtained by discrete time sampling from the continuous time model in

\footnotetext{
${ }^{5}$ In the case of Models A and C, in order to obtain ADF tests which are invariant to any serial correlation present in the driving shocks, Vogelsang and Perron (1989) show that the impulse dummy variable $1_{\left(t=t_{0}+1\right)}$ (and, where the ADF regression contains $p$ lagged dependent variables, $p$ lags of this impulse dummy) also needs to be included in the ADF regression. When using GLS detrending the impulse dummy $1_{\left(t=t_{0}+1\right)}$ (and lags thereof) does not need to be included in the second step ADF regression.

${ }^{6}$ Albeit for Models B and C, Kim and Perron (2009) show that the OLS levels estimator has to be trimmed around the estimate of the break date.
} 
(2.1)-(2.2). For the dynamic estimator of Hatanaka and Yamada (1999), the dummy variable $1_{(t=s+1)}$ already included in the estimated regression accounts for the interregnum term in (3.2).

Allowing for unnecessary broken intercept and trend variables in the unit root test specification leads to a loss of power to reject the unit root null when the data are stochastically stationary. As a consequence, pre-tests for the presence of breaks in the deterministic trend function that are robust to whether the series contains an autoregressive unit root or is stochastically stationary have been proposed; see inter alia, Harvey, Leybourne and Taylor (2007), Perron and Yabu (2009) and Sayginsoy and Vogelsang (2011). All of these pre-test methods are based on static regressions and so again will remain valid as formulated for data obtained by discrete time sampling from (2.1)-(2.2).

Finally, if we also allow the scale factor in (2.2) to display a one-time break then provided the heteroskedasticity-robust wild bootstrap implementations of the foregoing unit root test procedures, discussed in, for example, Cavaliere et al. (2011), are employed then these will remain valid without alteration for data obtained by discrete time sampling from (2.1)-(2.2). The large sample properties of the break fraction estimators and trend break pre-tests outlined above are unaffected.

\subsection{Methods Relating to Breaks in the Autoregressive Parameter}

Models allowing for deterministic changes in the autoregressive parameter have proved empirically useful in both applied macroeconomics where they provide a framework for testing for persistence change whereby a series admits a unit root in some periods but is mean reverting in other periods, and in empirical finance where they underlie testing procedures for the presence of rational explosive bubbles in price data.

A number of the methods proposed in the literature for persistence change testing and for detecting explosive price bubbles have been developed which are based on the same underlying statistical methodology which derives from the familiar ADF model where the autoregressive coefficient is allowed to display deterministic breaks. The former is typified by, inter alia, Banerjee et al. (1992), Leybourne et al. (1995) and Leybourne et al. (2007), and the latter by Phillips et al. (2011), Homm and Breitung (2012), Phillips et al. (2015), and Astill et al. (2017), inter alia. In these approaches a test is based not on a full sample ADF test statistic but rather on functions of sequences of subsample ADF statistics. Most commonly these sequences are based on either recursive subsamples, backward recursive subsamples, rolling subsamples, or rolling-recursive subsamples. In the case of persistence change, left-tailed tests are based on the smallest sub-sample ADF statistic in the computed sequence (i.e. the sub-sample ADF statistic which gives most weight to a stationary alternative). For bubble detection, right-tailed tests are based on the largest sub-sample ADF statistic (i.e. the sub-sample ADF statistic which gives most weight to an explosive alternative). In the persistence change tests, a linear trend tends to be allowed for, possibly with a level and/or trend break, while in the explosive bubbles literature an intercept is usually deemed sufficient.

It is clear from our results in Theorem 1 and Remarks 5 and 6 that, even without a one-time level or trend break, the coefficients on the interregnum term in (3.2) will differ from those in (3.1) and (3.3) in cases where the autoregressive parameter displays a one-time break that does not coincide 
with an observation point. ${ }^{7}$ As with the discussion in section 4.1, an implication of this is that for data obtained by discrete time sampling from the continuous time model in (2.1)-(2.2) the subsample ADF tests should be detrended (either by OLS or quasi-GLS) in levels (for the relevant subsample) rather than by including the deterministic regressors directly into the subsample ADF regression. The former is indeed done by Leybourne et al. (1995) and Leybourne et al. (2007) in the approaches they propose, but the latter is done by Banerjee et al. (1992), Phillips et al. (2011), Phillips et al. (2015) and Astill et al. (2017). Additionally, because the innovation variance differs across (3.1), (3.2) and (3.3) when either the autoregressive parameter or the scale factor in (2.3) displays a break, wild bootstrap implementations of the foregoing tests should be employed. Indeed, as Harvey et al. (2011,p.549) argue “... volatility changes in innovations to price series processes could be induced by the presence of a speculative bubble, but equally it could be the case that changes in volatility occur without an explosive bubble period occurring." For the case of the bubble detection test of Phillips et al. (2011), wild bootstrap implementations have been developed in Harvey et al. (2016), although like Phillips et al. (2011) they include the deterministic component in the subsample ADF regressions.

\section{Conclusions}

We have considered a simple model of deterministic one-time parameter change in a continuous time autoregressive model around a deterministic trend function. The exact discrete time analogue representation for this model was given and compared to extant parameter change models proposed in the discrete time literature. These were shown to coincide, excepting the observation immediately following the changepoint when the changepoint does not coincide with one of the discrete time observation points. The implication of these results for extant discrete time methods relating to models of one-time parameter change were discussed.

Although the continuous time model we have analysed in this paper is relatively simple, it nonetheless provides valuable insights into the properties of discrete time models of parameter change, providing a theoretical justification for a number of extant models of parameter change and statistical methods for discrete time data. Similar issues also arise in the temporal aggregation of discrete time models and so our results (suitably modified) have applicability beyond the temporal aggregation of continuous time models alone. Additionally, in empirical work using ADF regressions, it may be necessary to use additional lags in the regression to account for the observed serial correlation in the time series of interest. This can be achieved by considering a higher-order stochastic differential equation say of order $p$ - in (2.2), which would result in an autoregression in discrete time of order $p$. Some care would need to be taken, however, because, as pointed out by one of the referees, this would typically lead to $p$ interregnum points, rather than one. It is hoped that the results in this paper will encourage further research in this area.

\footnotetext{
${ }^{7}$ Empirical applications of bubble testing have tended to use monthly price data, so even here it seems, a priori, very unlikely that the break would happen to occur at an observation point.
} 


\section{A Appendix}

Proof of Theorem 1. For $t=1, \ldots, t_{0}, y(t)$ satisfies

$$
y(t)=\exp \left\{\rho_{0} t\right\} y(0)+\int_{0}^{t} \exp \left\{\rho_{0}(t-r)\right\}\left[\mu_{0}+\delta_{0} r\right] d r+\sigma_{0} \int_{0}^{t} \exp \left\{\rho_{0}(t-r)\right\} d B(r) .
$$

Evaluating at the point $t h$ and splitting the integrals over $(0, t h]$ into integrals over $(0, t h-h]$ and $(t h-h, t h]$ we find that $y_{t h}=c_{0, t h}+\phi_{0} y_{t h-h}+\eta_{0, t h}$, where

$$
c_{0, t h}=\int_{t h-h}^{t h} \exp \left\{\rho_{0}(t h-r)\right\}\left[\mu_{0}+\delta_{0} r\right] d r, \quad \eta_{0, t h}=\sigma_{0} \int_{t h-h}^{t h} \exp \left\{\rho_{0}(t h-r)\right\} d B(r) .
$$

By a change of variable $c_{0, t h}$ can be written

$$
\begin{aligned}
c_{0, t h} & =\int_{0}^{h} \exp \left\{\rho_{0} s\right\}\left[\mu_{0}+\delta_{0}(t h-s)\right] d s \\
& =\left(\int_{0}^{h} \exp \left\{\rho_{0} s\right\} d s\right) \mu_{0}+\left(\int_{0}^{h} \exp \left\{\rho_{0} s\right\} d s\right) \delta_{0} t h-\left(\int_{0}^{h} \exp \left\{\rho_{0} s\right\} s d s\right) \delta_{0},
\end{aligned}
$$

and evaluating these deterministic integrals yields $c_{0, t h}=c_{00}+c_{01} t h$. A similar procedure applies for $t=t_{1}+1, \ldots, N$ in which case $y(t)$ satisfies

$$
\begin{aligned}
y(t)= & \exp \left\{\left(\rho_{0}+\rho_{1}\right) t\right\} y(0)+\int_{0}^{t} \exp \left\{\left(\rho_{0}+\rho_{1}\right)(t-r)\right\}\left[\mu_{0}+\mu_{1}+\left(\delta_{0}+\delta_{1}\right) r\right] d r \\
& +\left(\sigma_{0}+\sigma_{1}\right) \int_{0}^{t} \exp \left\{\left(\rho_{0}+\rho_{1}\right)(t-r)\right\} d B(r) .
\end{aligned}
$$

This results in $y_{t h}=c_{1, t h}+\phi_{1} y_{t h-h}+\eta_{1, t h}$, where

$$
\begin{aligned}
c_{1, t h} & =\int_{t h-h}^{t h} \exp \left\{\left(\rho_{0}+\rho_{1}\right)(t h-r)\right\}\left[\mu_{0}+\mu_{1}+\left(\delta_{0}+\delta_{1}\right) r\right] d r, \\
\eta_{1, t h} & =\left(\sigma_{0}+\sigma_{1}\right) \int_{t h-h}^{t h} \exp \left\{\left(\rho_{0}+\rho_{1}\right)(t h-r)\right\} d B(r) .
\end{aligned}
$$

A similar change of variable and evaluation of the deterministic integrals yields $c_{1, t h}=c_{10}+c_{11} t h$.

It remains to determine the equation relating $y_{t_{1} h}$ to $y_{t_{0} h}$. We begin by relating $y_{t_{1} h}$ to the unobserved value of the process at the break point, $y(\tau T)$; we have, defining $\alpha_{1}=\rho_{0}+\rho_{1}$ for convenience,

$$
\begin{aligned}
y_{t_{1} h}= & \exp \left\{\alpha_{1}\left(t_{1} h-\tau T\right)\right\} y(\tau T)+\int_{\tau T}^{t_{1} h} \exp \left\{\alpha_{1}\left(t_{1} h-r\right)\right\}\left[\mu_{0}+\mu_{1}+\left(\delta_{0}+\delta_{1}\right) r\right] d r \\
& +\left(\sigma_{0}+\sigma_{1}\right) \int_{\tau T}^{t_{1} h} \exp \left\{\alpha_{1}\left(t_{1} h-r\right)\right\} d B(r) .
\end{aligned}
$$


Next we relate $y(\tau T)$ to the previous observation, $y_{t_{0} h}$, which yields

$$
\begin{aligned}
y(\tau T)= & \exp \left\{\rho_{0}\left(\tau T-t_{0} h\right)\right\} y_{t_{0} h}+\int_{t_{0} h}^{\tau T} \exp \left\{\rho_{0}(\tau T-r)\right\}\left(\mu_{0}+\delta_{0} r\right) d r \\
& +\sigma_{0} \int_{t_{0} h}^{\tau T} \exp \left\{\rho_{0}(\tau T-r)\right\} d B(r) .
\end{aligned}
$$

Substituting (A.2) into (A.1) yields an expression of the form $y_{t_{1} h}=c_{b, t_{1} h}+\phi_{b} y_{t_{0} h}+\eta_{b, t_{1} h}$, where

$$
\begin{aligned}
c_{b, t_{1} h}= & \int_{\tau T}^{t_{1} h} \exp \left\{\alpha_{1}\left(t_{1} h-r\right)\right\}\left[\mu_{0}+\mu_{1}+\left(\delta_{0}+\delta_{1}\right) r\right] d r \\
& +\exp \left\{\alpha_{1}\left(t_{1} h-\tau T\right)\right\} \int_{t_{0} h}^{\tau T} \exp \left\{\rho_{0}(\tau T-r)\right\}\left[\mu_{0}+\delta_{0} r\right] d r, \\
\phi_{b}= & \exp \left\{\alpha_{1}\left(t_{1} h-\tau T\right)\right\} \exp \left\{\rho_{0}\left(\tau T-t_{0} h\right)\right\}=\exp \left\{\rho_{0} h+\rho_{1}\left(t_{1} h-\tau T\right)\right\}, \\
\eta_{b, t_{1} h}= & \left(\sigma_{0}+\sigma_{1}\right) \int_{\tau T}^{t_{1} h} \exp \left\{\alpha_{1}\left(t_{1} h-r\right)\right\} d B(r) \\
& +\sigma_{0} \exp \left\{\alpha_{1}\left(t_{1} h-\tau T\right)\right\} \int_{t_{0} h}^{\tau T} \exp \left\{\rho_{0}(\tau T-r)\right\} d B(r) .
\end{aligned}
$$

Evaluation of the deterministic integral defining $c_{b, t_{1} h}$ yields the deterministic terms as required. Finally, the disturbances are individually and mutually serially uncorrelated as they are defined in terms of integrals of $d B(t)$ over non-overlapping intervals, while their variance properties follow by evaluating the relevant integrals.

\section{References}

Astill, S., Harvey, D.I., Leybourne, S.J. and Taylor, A.M.R. (2017). Tests for an end-of-sample bubble in financial time series. Econometric Reviews 36, 651-666.

Banerjee, A., Lumsdaine, R. and Stock, J.H. (1992). Recursive and sequential tests of the unit root and trend break hypothesis: theory and international evidence. Journal of Business and Economics Statistics 10, 271-287.

Bergstrom, A.R. (1984). Continuous time stochastic models and issues of aggregation over time. In: Griliches, Z. and Intriligator, M.D. (Eds.), Handbook of Econometrics (Volume 2). NorthHolland, Elsevier.

Carrion-i-Silvestre, J.L., Kim, D. and Perron, P. (2009). GLS-based unit root tests with multiple structural breaks under both the null and the alternative hypotheses. Econometric Theory 25, $1754-1792$.

Chambers, M.J. (2015). Testing for a unit root in a near-integrated model with skip-sampled data. Journal of Time Series Analysis 36, 630-649.

Cavaliere, G., Harvey, D.I., Leybourne, S.J. and Taylor, A.M.R. (2011). Testing for unit roots in the presence of a possible break in trend and non-stationary volatility. Econometric Theory 27, 957-991. 
Harris, D., Harvey, D.I., Leybourne, S.J. and Taylor, A.M.R. (2009). Testing for a unit root in the presence of a possible break in trend. Econometric Theory 25, 1545-1588.

Harvey, D.I., Leybourne, S. J., Sollis, R., Taylor, A.M.R. (2016). Tests for explosive financial bubbles in the presence of non-stationary volatility. Journal of Empirical Finance 38, 548-574.

Hatanaka, M. and Yamada, K. (1999). A unit root test in the presence of structural changes in I(1) and I(0) models. In: Engle, R.F. and White, H. (Eds.), Cointegration, and Forecasting: A Festschrift in Honour of Clive W. J. Granger. Oxford University Press.

Homm, U. and Breitung, J. (2012). Testing for speculative bubbles in stock markets: a comparison of alternative methods. Journal of Financial Econometrics 10, 198-231.

Kim, D. and Perron, P. (2009). Unit root tests allowing for a break in the trend function under both the null and alternative hypotheses. Journal of Econometrics 148, 1-13.

Kim, J.Y. (2000). Detection of change in persistence of a linear times series. Journal of Econometrics 95, $97-116$.

Leybourne, S., Kim, T., Smith, V. and Newbold, P. (2003). Tests for a change in persistence against the null of difference-stationarity. Econometrics Journal 6, 291-311.

Leybourne, S., Kim, T.-H. and Taylor, A.M.R. (2007). Detecting multiple changes in persistence. Studies in Non-Linear Dynamics and Econometrics 11:3, Article 2.

Perron, P. (1989). The great crash, the oil price shock, and the unit root hypothesis. Econometrica 57, 1361-1401.

Perron, P. (1997). Further evidence of breaking trend functions in macroeconomic variables. Journal of Econometrics 80, 355-385.

Perron, P. and Rodríguez, G. (2003). GLS detrending, efficient unit root tests and structural change. Journal of Econometrics 115, 1-27.

Perron, P. and Yabu, T. (2009). Testing for shifts in trend with an integrated or stationary noise component. Journal of Business and Economic Statistics 27, 369-396.

Perron, P. and Zhu, X. (2005). Structural breaks with deterministic and stochastic trends. Journal of Econometrics 129, 65-119.

Phillips, P.C.B., Shi, S.-P. and Yu, J. (2015). Testing for multiple bubbles: historical episodes of exuberance and collapse in the S\&P 500. International Economic Review 56, 1043-1078.

Phillips, P.C.B., Wu, Y. and Yu, J. (2011). Explosive behavior in the 1990s Nasdaq: when did exuberance escalate stock values? International Economic Review 52, 201-226.

Vogelsang, T.J. and Perron, P. (1998). Additional tests for a unit root allowing the possibility of breaks in the trend function. International Economic Review 39, 1073-1100.

Zivot, E. and Andrews, D.W.K. (1992). Further evidence on the great crash, the oil-price shock, and the unit-root hypothesis. Journal of Business and Economic Statistics 10, 251-270. 